HD-THEP-97-28

SOGANG-HEP 222/97

\title{
Hamiltonian Embedding of SU(2) Higgs Model in the Unitary Gauge
}

\author{
Yong-Wan Kim円, Young-Jai Park ${ }^{1}$ and Klaus D. Rothe? \\ Institut für Theoretische Physik \\ Universität Heidelberg \\ Philosophenweg 16, D-69120 Heidelberg
}

\begin{abstract}
Following systematically the generalized Hamiltonian approach of Batalin, Fradkin and Tyutin (BFT), we embed the second-class non-abelian SU(2) Higgs model in the unitary gauge into a gauge invariant theory. The strongly involutive Hamiltonian and constraints are obtained as an infinite power series in the auxiliary fields. Furthermore, comparing these results with those obtained from the gauged second class Lagrangian, we arrive at a simple interpretation for the first class Hamiltonian, constraints and observables.
\end{abstract}

PACS: 11.10.Ef, 11.10.Kk, 11.15.-q

Keywords: Hamiltonian embedding; Second class; SU(2); Higgs

\footnotetext{
${ }^{1}$ e-mail: kim@thphys.uni-heidelberg.de and yjpark@ccs.sogang.ac.kr

On leave of absence from Department of Physics and Basic Science Research Institute, Sogang University, C.P.O.Box 1142, Seoul 100-611, Korea

2e-mail: k.rothe@thphys.uni-heidelberg.de
} 


\section{Introduction}

The quantization of second-class Hamiltonian systems [1] requires the strong implementation of the second-class constraints. This may imply Dirac brackets, whose non-canonical structure may pose problems on operator level. This makes it desirable to embed the second-class theory into a first-class one, where the commutator relations remain canonical and the constraints are imposed on the states. An example is provided by the Higgs model with spontaneous symmetry breakdown [2] whose quantization is usually carried out in the so called "unitary" gauge. As is well known, in this gauge the model is a purely second class system characterized by two sets of second class constraints [3, 4]. The required strong implementation of these constraints leads to non-polynomial field dependent Dirac brackets. As mentioned above, one can circumvent the problems associated with this non-polynomial dependence by turning this system into a first class one with a usual Poisson bracket structure in an extended phase space and implementing the first class constraints on the physical states. A systematic procedure for achieving this has been given by Batalin and Fradkin (BF) [5] and has been explicitly carried out for the above model for the abelian case [4]. The construction in the BF framework proved already non-trivial in the abelian case, and does not appear particularly suited for treating the non-abelian case.

In this paper we shall generalize the above program to the case of the non-abelian SU(2) Higgs model as a nontrivial and simple example by following a simpler constructive procedure based on modified version of the BF-formalism, due to Batalin and Tyutin [6]. This procedure has been recently applied to several interesting abelian [7, 8, 9] and non-abelian models [10, 11, 12], and will also render the analysis of the $\mathrm{SU}(2)$ Higgs model in the unitary gauge more transparent.

Starting from the second class Lagrangian, we systematically construct in section 2 the corresponding first class, strongly involutive constraints, following the BFTprocedure. In section 3 we then use the same procedure in order to construct the 
first-class fields in strong involution with the constraints. The first class Hamiltonian is obtained from the original second-class Hamiltonian by replacing the original fields by the corresponding first class ones. This Hamiltonian is thus again in strong involution with the constraints. In section 4 we then show that the results coincide with those obtained by gauging the original second class Lagrangian, after a suitable canonical transformation. This establishes the equivalence of the BFT construction and the Lagrangian quantization procedure based on the addition of a Wess-Zumino (WZ) term [13, 14, 15] (see also [10, 11, 12]). We conclude in section 5 with a summary.

\section{BFT construction of first class constraints}

Consider the non-Abelian SU(2) Higgs model in the unitary gauge,

$$
\mathcal{L}\left(B^{\mu}, \eta\right)=\frac{1}{4} \operatorname{tr} G_{\mu \nu} G^{\mu \nu}-\frac{1}{2} m^{2}(\eta) \operatorname{tr} B_{\mu} B^{\mu}+\frac{1}{2} \partial_{\mu} \eta \partial^{\mu} \eta+V(\eta)
$$

where $V(\eta)$ is the Higgs potential

$$
V(\eta)=\frac{\mu^{2}}{2}(\eta+v)^{2}-\frac{\lambda}{4}(\eta+v)^{4}
$$

with the vacuum expectation value $v$, and for later convenience we define the field dependent mass squared $m^{2}(\eta)$ as follows

$$
m^{2}(\eta)=(\eta+v)^{2}
$$

Here the vector potential $B^{\mu}$ is an antihermitian Lie algebra valued field

$$
B^{\mu}=t^{a} B^{\mu a} \quad(a=1,2,3)
$$

where $t^{a}$ are the $\mathrm{SU}(2)$ group generators, and $G^{\mu \nu}$ is the associated field strength tensor

$$
G^{\mu \nu}=\partial^{\mu} B^{\nu}-\partial^{\nu} B^{\mu}+\left[B^{\mu}, B^{\nu}\right]
$$


Our conventions are

$$
\left[t^{a}, t^{b}\right]=\epsilon^{a b c} t^{c}, \quad \operatorname{tr}\left(t^{a} t^{b}\right)=-\delta^{a b}
$$

where $\epsilon^{a b c}$ is the structure constant of the $\mathrm{SU}(2)$ group. The momenta canonically conjugate to $B^{0 a}, B^{i a}$ and $\eta$ are respectively given by $\pi_{0}^{a}=0, \pi_{i}^{a}=G_{i 0}^{a}$ and $\pi=\dot{\eta}$. We thus have the primary constraints $\pi_{0}^{a}=0$. The canonical Hamiltonian density associated with the Lagrangian (2.1) is found to be

$\mathcal{H}_{C}=\frac{1}{2}\left(\pi_{i}^{a}\right)^{2}+\frac{1}{2} \pi^{2}+\frac{1}{4}\left(G_{i j}^{a}\right)^{2}+\frac{1}{2} m^{2}(\eta)\left(B^{i a}\right)^{2}+\frac{1}{2} m^{2}(\eta)\left(B^{0 a}\right)^{2}+\frac{1}{2}\left(\partial_{i} \eta\right)^{2}-V(\eta)-B^{0 a} \Omega_{2}^{a}$.

Since persistency in time of these constraints leads to further (secondary) constraints $\Omega_{2}^{a}=0$, this system is described by two sets of second class constraints:

$$
\begin{aligned}
& \Omega_{1}^{a}=\pi_{0}^{a}, \\
& \Omega_{2}^{a}=\left(D^{i} \pi_{i}\right)^{a}+m^{2}(\eta) B^{0 a},
\end{aligned}
$$

where the covariant derivative is given by $\left(D^{i} \pi_{i}\right)^{a}=\partial^{i} \pi_{i}^{a}+\epsilon^{a b c} B^{i b} \pi_{i}^{c}$. Then, the constraints fully form the second class algebra as follows

$$
\left\{\Omega_{i}^{a}(x), \Omega_{j}^{b}(y)\right\}=\Delta_{i j}^{a b}(x, y)
$$

with

$$
\Delta_{i j}^{a b}(x, y)=\left(\begin{array}{cc}
0 & -m^{2}(\eta) \delta^{a b} \\
m^{2}(\eta) \delta^{a b} & \epsilon^{a b c}\left(D^{k} \pi_{k}\right)^{c}
\end{array}\right) \delta^{3}(x-y) .
$$

We now convert the second class system defined by the "commutation relations" (2.9) to a first-class system at the expense of introducing additional degrees of freedom. Following refs. [5, 6], we introduce auxiliary fields $\Phi^{1 a}$ and $\Phi^{2 a}$ corresponding to $\Omega_{1}^{a}$ and $\Omega_{2}^{a}$, with the Poisson bracket

$$
\left\{\Phi^{i a}(x), \Phi^{j b}(y)\right\}=\omega_{a b}^{i j}(x, y),
$$


where we are free [5] to make the choice

$$
\omega_{a b}^{i j}(x, y)=\epsilon^{i j} \delta_{a b} \delta^{3}(x-y) .
$$

The first class constraints $\tilde{\Omega}_{i}^{a}$ are now constructed as a power series in the auxiliary fields,

$$
\tilde{\Omega}_{i}^{a}=\Omega_{i}^{a}+\sum_{n=1}^{\infty} \Omega_{i}^{(n) a},
$$

where $\Omega_{i}^{(n) a}(n=1, \ldots, \infty)$ are homogeneous polynomials in the auxiliary fields $\left\{\Phi^{j b}\right\}$ of degree $n$, to be determined by the requirement that the constraints $\tilde{\Omega}_{i}^{a}$ be strongly involutive:

$$
\left\{\tilde{\Omega}_{i}^{a}(x), \tilde{\Omega}_{j}^{b}(y)\right\}=0 .
$$

Making the ansatz

$$
\Omega_{i}^{(1) a}(x)=\int d^{3} y X_{i j}^{a b}(x, y) \Phi^{j b}(y)
$$

and substituting (2.15) into (2.14) leads to the condition

$$
\int d^{3} z d^{3} z^{\prime} X_{i k}^{a c}(x, z) \omega_{c d}^{k l}\left(z, z^{\prime}\right) X_{j \ell}^{b d}\left(z^{\prime}, y\right)=-\Delta_{i j}^{a b}(x, y) .
$$

With the choice (2.12) for $\omega_{a b}^{i j}(x, y)$, Eq. (2.16) has (up to a natural arbitrariness) the solution

$$
X_{i j}^{a b}(x, y)=\left(\begin{array}{cc}
m^{2}(\eta) \delta^{a b} & 0 \\
-\frac{1}{2} \epsilon^{a b c}\left(\mathcal{D}^{k} \pi_{k}\right)^{c} & \delta^{a b}
\end{array}\right) \delta^{3}(x-y) .
$$

From the symplectic structure of Eq. (2.11) with the choice (2.12) for $\omega_{a b}^{i j}(x, y)$, we may identify the auxiliary fields with canonically conjugated pairs. We make this explicit by adopting the notation

$$
\left(\Phi^{1 a}, \Phi^{2 a}\right) \Rightarrow\left(\theta^{a}, \pi_{\theta}^{a}\right)
$$


Substituting (2.17) into (2.15) as well as (2.13), and iterating this procedure one finds the strongly involutive first class constraints to be given by

$$
\begin{aligned}
& \tilde{\Omega}_{1}^{a}=\pi_{0}^{a}+m^{2}(\eta) \theta^{a}, \\
& \tilde{\Omega}_{2}^{a}=m^{2}(\eta) B^{0 a}+V^{a b}(\theta)\left(\mathcal{D}^{i} \pi_{i}\right)^{b}+\pi_{\theta}^{a} .
\end{aligned}
$$

Here

$$
(a d \theta)^{a b}=\epsilon^{a c b} \theta^{c}
$$

and

$$
V(\theta)=\sum_{n=0}^{\infty} \frac{(-1)^{n}}{(n+1) !}(\operatorname{ad} \theta)^{n}
$$

where $a d \theta$ denotes the Lie algebra valued field $\theta$ in the adjoint representation, ad $\theta=\theta^{a} T^{a}$, with $T_{a b}^{c}=\epsilon^{a c b}$. This completes the construction of the first class constraints.

\section{First class fields and Hamiltonian}

Let $\mathcal{J}$ denote collectively the variables $\left(B^{\mu a}, \pi_{\mu}^{a}, \eta, \pi\right)$ of the original phase space. The construction of the first class Hamiltonian $\tilde{H}$ can be done along similar lines as in the case of the constraints, by representing it as a power series in the auxiliary fields and requiring $\left\{\tilde{\Omega}_{i}^{a}, \tilde{H}\right\}=0$ subject to the condition $\tilde{H}\left[\mathcal{J}, \theta^{a}=\pi_{\theta}^{a}=0\right]=H_{C}$. We shall follow here a somewhat different path [9, 10, 11, 12] by noting that any functional of first class fields $\tilde{\mathcal{J}}$ corresponding to $\mathcal{J}$ in the extended phase space will also be first class. This leads us to the identification $\tilde{H}=H_{C}[\tilde{\mathcal{J}}]$. The "physical" fields $\tilde{\mathcal{J}}$ are obtained as a power series in the auxiliary fields $\left(\theta^{a}, \pi_{\theta}^{a}\right)$ by requiring them to be strongly involutive: $\left\{\tilde{\Omega}_{i}^{a}, \tilde{\mathcal{J}}\right\}=0$. The iterative solution of these equations involves the use of (2.12) and (2.17) and leads to an infinite series which can be compactly written in terms of $a d \theta$ defined in (2.19) as

$$
\begin{aligned}
\tilde{B}^{0 a} & =B^{0 a}+\frac{1}{m^{2}(\eta)} \pi_{\theta}^{a}-\frac{1}{m^{2}(\eta)}\left(U^{a b}(\theta)-V^{a b}(\theta)\right)\left(\mathcal{D}^{i} \pi_{i}\right)^{b} \\
\tilde{B}^{i a} & =U^{a b}(\theta) B^{i b}+V^{a b}(\theta) \partial^{i} \theta^{b}
\end{aligned}
$$




$$
\begin{aligned}
\tilde{\pi}_{0}^{a} & =\pi_{0}^{a}+m^{2}(\eta) \theta^{a}, \\
\tilde{\pi}_{i}^{a} & =U^{a b}(\theta) \pi_{i}^{b}, \\
\tilde{\eta} & =\eta, \\
\tilde{\pi} & =\pi+2 m(\eta) B^{0 a} \theta^{a},
\end{aligned}
$$

where $V(\theta)$ has been defined in (2.20) and $U(\theta)$ is given by

$$
U(\theta)=\sum_{n=0}^{\infty} \frac{(-1)^{n}}{n !}(a d \theta)^{n}=e^{-a d \theta} .
$$

We now observe that the first class constraints (2.18) can also be written in terms of the physical fields as

$$
\begin{aligned}
\tilde{\Omega}_{1}^{a} & =\tilde{\pi}_{0}^{a}, \\
\tilde{\Omega}_{2}^{a} & =\left(\widetilde{\mathcal{D}^{i} \pi_{i}}\right)^{a}+m^{2}(\eta) \tilde{B}^{0 a} .
\end{aligned}
$$

Comparing with the second class constraints $\Omega_{i}^{a}$ in Eq. (2.8), we see that the first class constraints (3.3) are just the second class constraints written in terms of the physical variables. Correspondingly, we take the first class Hamiltonian density $\tilde{\mathcal{H}}_{C}$ to be given by the second class one (2.7), expressed in terms of the physical fields:

$\tilde{\mathcal{H}}_{C}=\frac{1}{2}\left(\tilde{\pi}_{i}^{a}\right)^{2}+\frac{1}{2}(\tilde{\pi})^{2}+\frac{1}{4}\left(\tilde{G}_{i j}^{a}\right)^{2}+\frac{1}{2} m^{2}(\tilde{\eta})\left(\tilde{B}^{i a}\right)^{2}+\frac{1}{2} m^{2}(\tilde{\eta})\left(\tilde{B}^{0 a}\right)^{2}+\frac{1}{2}\left(\partial_{i} \tilde{\eta}\right)^{2}-V(\tilde{\eta})-\tilde{B}^{0 a} \tilde{\Omega}_{2}^{a}$.

It is important to notice that any Hamiltonian weakly equivalent to (3.4) describes the same physics since the observables of the first class formulation must be first class themselves. Hence we are free to add to $\tilde{\mathcal{H}}$ any terms proportional to the first class constraints.

For later comparison, we explicitly rewrite the above Hamiltonian by making use of the expressions in Eq. (3.1):

$$
\begin{aligned}
\tilde{\mathcal{H}}_{C} & =\frac{1}{2}\left(\pi_{i}^{a}\right)^{2}+\frac{1}{2}\left(\pi+2 m(\eta) B^{0 a} \theta^{a}\right)^{2}+\frac{1}{4}\left(G_{i j}^{a}\right)^{2}+\frac{1}{2} m^{2}(\eta)\left(U^{a b}(\theta) B^{i b}+V^{a b}(\theta) \partial^{i} \theta^{b}\right)^{2} \\
& +\frac{1}{2}\left(\partial_{i} \eta\right)^{2}-V(\eta)+\frac{1}{2 m^{2}(\eta)}\left(\left(\mathcal{D}^{i} \pi_{i}\right)^{a}\right)^{2}-\frac{1}{2 m^{2}(\eta)}\left(\tilde{\Omega}_{2}^{a}\right)^{2} .
\end{aligned}
$$




\section{Lagrangian interpretation of the results}

Let us first define the group valued field

$$
g(\theta)=e^{\theta}, \quad \theta=\theta^{a} t^{a}
$$

Then, we have for a Lie algebra valued field $A=A^{a} t^{a}$,

$$
\begin{gathered}
-\operatorname{tr}\left(t^{a} g^{-1}(\theta) A g(\theta)\right)=U^{a b}(\theta) A^{b} \\
-\operatorname{tr}\left(t^{a} g^{-1}(\theta) \partial_{\mu} g(\theta)\right)=V^{a b}(\theta) \partial_{\mu} \theta^{b}
\end{gathered}
$$

where the r.h.s. resumes in compact form an infinite series given by Eqs. (2.20) and (3.2). However, since the time component fields $\tilde{B}^{0 a}$ in Eq. (3.1) or Eq. (3.3) can be at most rewritten as

$$
\tilde{B}^{0 a}=\frac{1}{m^{2}(\eta)}\left(\tilde{\Omega}_{2}^{a}-U^{a b}(\theta)\left(\mathcal{D}^{i} \pi_{i}\right)^{b}\right)
$$

it is still difficult to directly understand the strong relation of the gauge transform. On the other hand, the spatial component fields $\tilde{B}^{i a}$ have a simple interpretation. From (4.2) we see that the field $\tilde{B}^{i a}$ in the expression (3.1) can be written in compact form as

$$
\tilde{B}^{i}=g^{-1} B^{i} g+g^{-1} \partial^{i} g
$$

which shows that $\tilde{B}^{i}$ is just the gauge transform of $B^{i}$. This suggests that as in the case of the non-abelian self-dual model [12], our BFT results should be equivalent to the ones obtained by gauging $B^{\mu}$ in the Lagrangian (2.1). We now show that this is indeed the case.

Gauging the Lagrangian (2.1) by making the substitution

$$
B^{\mu} \rightarrow \hat{B}^{\mu}=g^{-1} B^{\mu} g+g^{-1} \partial^{\mu} g
$$

we obtain

$$
\hat{\mathcal{L}}(B, \eta, g)=\mathcal{L}(B, \eta)+\mathcal{L}_{W Z}
$$


where

$$
\mathcal{L}_{W Z}(B, \eta, g)=-m^{2}(\eta)\left(\operatorname{tr}\left(B^{\mu} \partial_{\mu} g g^{-1}\right)+\frac{1}{2} \operatorname{tr}\left(g^{-1} \partial^{\mu} g\right)^{2}\right)
$$

plays the role of the Wess-Zumino-Witten (WZW) term in the gauge-invariant formulation of two-dimensional chiral gauge theories [13, [14, [15], and $\mathcal{L}(B, \eta)$ is the Lagrangian of the second class system.

We then have for the momentum $\Pi$ conjugate to $g$,

$$
\Pi^{T}=-m^{2}(\eta)\left(g^{-1} B^{0}+g^{-1} \partial^{0} g g^{-1}\right),
$$

where " $T$ " denotes "transpose". The other canonical momenta $\pi_{\mu}$ and $\pi$ are the same as before. Hence the primary constraints are still of the form in Eq. (2.8), $T_{1}^{a}=\pi_{0}^{a}$, though the dynamics is a different one. The canonical Hamiltonian corresponding to (3.5) then reads, on the constraint surface $\pi_{0}^{a}=0$,

$$
\begin{aligned}
\hat{\mathcal{H}}_{C}= & \frac{1}{2}\left(\pi_{i}^{a}\right)^{2}+\frac{1}{2} \pi^{2}-\frac{1}{4} \operatorname{tr}\left(G_{i j}\right)^{2}+\frac{1}{2} m^{2}(\eta)\left(B^{i a}\right)^{2} \\
& +m^{2}(\eta) \operatorname{tr}\left(B^{i} \partial_{i} g g^{-1}\right)-\frac{1}{2} m^{2}(\eta) \operatorname{tr}\left(g^{-1} \partial_{i} g\right)^{2}+\frac{1}{2}\left(\partial_{i} \eta\right)^{2}-V(\eta) \\
& -\frac{1}{2 m^{2}(\eta)} \operatorname{tr}\left(\Pi^{T} g\right)^{2}+\operatorname{tr} B^{0}\left(\mathcal{D}^{i} \pi_{i}-g \Pi^{T}\right) .
\end{aligned}
$$

From (4.9) we see that persistency in time of the primary constraints $\pi_{0}^{a}=0$ implies secondary constraints associated with the Lagrange multipliers $B^{0 a}$. We thus have two sets of first class constraints:

$$
\begin{aligned}
& T_{1}^{a}=\pi_{0}^{a} \approx 0, \\
& T_{2}^{a}=\left(\mathcal{D}^{i} \pi_{i}\right)^{a}+\operatorname{tr}\left(t^{a} g \Pi^{T}\right) \approx 0 .
\end{aligned}
$$

In order to establish the connection with the BFT results in section 3, we must expand the terms involving $g$ and $\Pi^{T}$ into an infinite series in the field $\theta$ using the identities (4.2) and the following properties of the Lie algebra and group valued functions, $V(\theta)$ and $U(\theta)$ defined in Eqs. (2.20) and (3.2), respectively:

$$
\begin{aligned}
V^{a b}(-\theta)= & V^{b a}(\theta), \quad U^{a b}(-\theta)=U^{b a}(\theta), \\
U^{c a}(\theta) V^{c b}(\theta)= & V^{a b}(-\theta), \quad U^{a c}(\theta) V^{b c}(\theta)=V^{a b}(\theta), \\
& U^{c a}(\theta) U^{c b}(\theta)=U^{a c}(\theta) U^{b c}(\theta)=\delta^{a b} .
\end{aligned}
$$


The WZ term (4.7) can then be rewritten in form

$$
\mathcal{L}_{W Z}=m^{2}(\eta) B^{\mu a} U^{c a}(\theta) V^{c b}(\theta) \partial_{\mu} \theta^{b}+\frac{1}{2} m^{2}(\eta) V^{c a}(\theta) V^{c b}(\theta) \partial_{\mu} \theta^{a} \partial^{\mu} \theta^{b} .
$$

From here we obtain for the momenta canonically conjugate to $\theta^{a}$

$$
\pi_{\theta}^{\prime a}=m^{2}(\eta) \hat{B}^{0 c} V^{c a}(\theta)
$$

where

$$
\hat{B}^{0 c}=U^{c b}(\theta) B^{0 b}+V^{c b}(\theta) \partial^{0} \theta^{b}
$$

is readily identified with the zero component of the gauged vector potential (4.5), and where we used the "prime" on $\pi_{\theta}^{a}$ in order to distinguish this momentum from the auxiliary field $\pi_{\theta}^{a}$ in Eq. (2.18) introduced in the BFT construction in section 2.

It remains to establish the relation with the results of section 3. Multiplying (4.8) from the left with $g$ and using (4.2) we have

$$
\begin{aligned}
\operatorname{tr}\left(t^{a} g \Pi^{T}\right) & =m^{2}(\eta)\left(B^{0 a}+V^{a b}(-\theta) \partial^{0} \theta^{b}\right) \\
& =m^{2}(\eta)\left(B^{0 a}+U^{c a}(\theta) V^{c b}(\theta) \partial^{0} \theta^{b}\right) .
\end{aligned}
$$

Making use of (4.14), (4.11) and (4.10), we obtain from (4.15),

$$
\hat{B}^{0 a}=\frac{1}{m^{2}(\eta)} U^{a b}(\theta) \operatorname{tr}\left(t^{b} g \Pi^{T}\right)=\frac{1}{m^{2}(\eta)} U^{a b}(\theta)\left(T_{2}-\mathcal{D}^{i} \pi_{i}\right)^{b} .
$$

Comparing this with (4.3), we conclude $\hat{B}^{0 a} \approx \tilde{B}^{0 a}$ since $\hat{B}^{0 a}$ and $\tilde{B}^{0 a}$ are identical up to additive terms proportional to the first class constraints. This establishes the weak equivalence of $\hat{B}^{\mu a}$ and $\tilde{B}^{\mu a}$. Furthermore, combining (4.16) and (4.13) we have

$$
\pi_{\theta}^{\prime a}=U^{a c}(\theta) V^{b c}(\theta) \operatorname{tr}\left(t^{b} g \Pi^{T}\right) .
$$

Using (4.10) we rewrite this as

$$
V^{a b}(\theta) T_{2}^{b}=\pi_{\theta}^{\prime a}+V^{a b}(\theta)\left(\mathcal{D}^{i} \pi_{i}\right)^{b}
$$


Performing the canonical transformation

$$
\begin{aligned}
\pi_{0}^{a} & \rightarrow \pi_{0}^{a}+m^{2}(\eta) \theta^{a} \\
\pi_{\theta}^{\prime a} & \rightarrow \pi_{\theta}^{\prime a}+m^{2}(\eta) B^{0 a} \\
\pi & \rightarrow \pi+2 m(\eta) B^{0 a} \theta^{a}
\end{aligned}
$$

we see that the first class constraints $T_{1}^{a}=\pi_{0}^{a} \approx 0$ and $V^{a b}(\theta) T_{2}^{b} \approx 0$ map into the constraints (2.18) in the BFT construction.

It now remains to check the relation between $\hat{\mathcal{H}}_{C}$ and $\tilde{\mathcal{H}}_{C}$ as given by (4.9) and (3.5), respectively. Making use of (4.14) and (4.19), expression (4.9) for $\hat{\mathcal{H}}_{C}$ may be rewritten in the following form in order to compare with $\tilde{\mathcal{H}}_{C}$ in Eq. (3.5)

$$
\begin{aligned}
\hat{\mathcal{H}}_{C} & =\frac{1}{2}\left(\pi_{i}^{a}\right)^{2}+\frac{1}{2}\left(\pi+2 m(\eta) B^{0 a} \theta^{a}\right)^{2}+\frac{1}{4}\left(G_{i j}^{a}\right)^{2}+\frac{1}{2} m^{2}(\eta)\left(U^{a b}(\theta) B^{i b}+V^{a b}(\theta) \partial^{i} \theta^{b}\right)^{2} \\
& +\frac{1}{2}\left(\partial_{i} \eta\right)^{2}-V(\eta)+\frac{1}{2 m^{2}(\eta)}\left(\left(\mathcal{D}^{i} \pi_{i}\right)^{a}\right)^{2}+\frac{1}{2 m^{2}(\eta)}\left(T_{2}^{a}\right)^{2} \\
& -\frac{1}{m^{2}(\eta)}\left(m^{2}(\eta) B^{0 a}+\left(\mathcal{D}^{i} \pi_{i}\right)^{a}\right) T_{2}^{a} .
\end{aligned}
$$

Then, we immediately obtain the equivalence relation $\hat{\mathcal{H}}_{C} \approx \tilde{\mathcal{H}}_{C}$ since $\hat{\mathcal{H}}_{C}$ is identical with $\tilde{\mathcal{H}}_{C}$ up to additive terms proportional to the first class constraints. We have thus arrived at a simple interpretation of the results obtained in section 3 .

Let us compare the Hamiltonian (3.5) with the one given in Eq. (2.29) of [4]. Making use of (2.18) we may rewrite $\tilde{\mathcal{H}}_{C}$ in the form

$$
\tilde{\mathcal{H}}_{C}=\mathcal{H}_{C}+\Delta \mathcal{H}
$$

where $\mathcal{H}_{C}$ has been defined in (2.7) and $\Delta \mathcal{H}$ is given by

$$
\begin{aligned}
\Delta \mathcal{H}_{C} & =2 m(\eta) B^{0 a} \theta^{a}\left(\pi+m(\eta) B^{0 b} \theta^{b}\right)+m^{2}(\eta) V^{a b}(\theta) \partial^{i} \theta^{b}\left(U^{a c}(\theta) B^{i c}+\frac{1}{2} V^{a c}(\theta) \partial^{i} \theta^{c}\right) \\
& +\frac{1}{2 m^{2}(\eta)}\left(\tilde{\Omega}_{2}^{a}-\pi_{\theta}^{a}+\left(\delta^{a b}-V^{a b}(\theta)\right)\left(\mathcal{D}^{i} \pi_{i}\right)^{b}\right)-\frac{1}{2 m^{2}(\eta)}\left(\tilde{\Omega}_{2}^{a}\right)^{2} .
\end{aligned}
$$

Taking the abelian limit, and comparing (4.21) with the Hamiltonian (2.29) of [4] one sees that the two Hamiltonians are weakly equal. 
The quantization of the first class system now proceeds following a standard procedure [16] involving the construction of the BRST Hamiltonian $H_{B R S T}$ as well as the unitarizing Hamiltonian $H_{U}$ obtained from $H_{B R S T}$ by the addition of a BRST exact term containing the gauge fixing (fermionic) function. The steps are analogous to those taken in the abelian case of $\mathbb{4}$, the only difference being that in the present case the algebra of the first class constraints and Hamiltonian is strongly involutive, so that the BRST Hamiltonian coincides with the first class Hamiltonian $\tilde{\mathcal{H}}_{C}$ in $(3.5)$. In this respect the situation here differs from that of the abelian case discussed in [由], where the first class Hamiltonian $H^{\prime}$ is in weak involution with the constraint $\tilde{\Omega}_{1}$. However, the resulting difference in the unitarizing Hamiltonian can be shown not to affect the corresponding path integral once part of the integration over the ghosts has been performed. The recovery of the Higgs model in the unitary gauge for the choice $\chi_{1}^{a}=B^{0 a}, \chi_{2}^{a}=\theta^{a}$ of the gauge fixing functions, as well as its usual gauge invariant formulation in terms of a complex scalar doublet $\phi=g(\theta) \frac{1}{\sqrt{2}}(0, \eta+v)$ (with the BFT fields $\theta^{a}$ playing the role of the Goldstone boson) in the FaddeevPopov type gauges $\chi_{1}^{a}=B^{0 a}$ and $\chi_{2}^{a}=f\left(B^{i}\right)$, thus proceeds along the lines of the discussion of [4].

\section{Conclusion}

The main objective of this paper was to provide a nontrivial, physically interesting example for the Hamiltonian embedding of a second-class theory into a first-class one, following the systematic constructive procedure of Batalin, Fradkin, and Tyutin [5], 6]. Unlike the case of the abelian models discussed in the literature, the first class Hamiltonian and secondary constraints generated by this procedure are found to be given by an infinite power series in the auxiliary fields in the extended phase space. By explicitly summing this series we established the weak equivalence with the corresponding quantities as obtained by gauging the second class Lagrangian defining our $\mathrm{SU}(2)$ Higgs model in the unitary gauge, with the auxiliary fields $\theta^{a}$ 
playing the role of the corresponding gauge degrees of freedom. We thereby showed that on the space of gauge-invariant functionals the Lagrangian approach of refs. [13, 14] for embedding second class theories into a gauge theory is equivalent to the Hamiltonian BFT approach. To do this we used the most economical way for obtaining the desired results, by working with group rather than Lie algebra valued fields in the gauged Lagrangian.

\section{Acknowledgment}

Two of the authors (Y.-W. Kim and Y.-J. Park) would like to thank the Institut für Theoretische Physik for their warm hospitality. The present study was partly supported by the Korea Research Foundation for (1996) overseas fellowship and the DFG-KOSEF Exchange Program, which made this collaboration possible.

\section{References}

[1] P. A. M. Dirac, Can. J. Math. 2 (1950) 129: "Lectures on Quantum Mechanics" (Yeshiva University, New York 1964).

[2] E. S. Abers and B. W. Lee, Phys. Rep. C9 (1973) 1.

[3] H. O. Girotti and H. J. Rothe, Nuovo Cim, 75 A (1983) 62.

[4] R. Banerjee, H. J. Rothe and K. D. Rothe, Nucl. Phys. B426 (1994) 129.

[5] I. A. Batalin and E. S. Fradkin, Phys. Lett. B180 (1986) 157; Nucl. Phys. B279 (1987) 514.

[6] I. A. Batalin and I. V. Tyutin, Int. J. Mod. Phys. A6 (1991) 3255.

[7] R. Banerjee and H. J. Rothe, Nucl. Phys. B447 (1995) 183; Y.-W. Kim, Y.-J. Park, K. Y. Kim and Y. Kim, Phys. Rev. D51 (1995) 2943; J.-H. Cha, Y.W. Kim, Y.-J. Park, Y. Kim and W. T. Kim, Z. Phys. C69 (1995) 175; N. 
Banerjee, R. Banerjee and S. Ghosh, Ann. Phys. (NY) 241 (1995) 237; R. J. Banerjee, H. J. Rothe and K. D. Rothe, Phys. Rev. D52 (1995) 3750; ibid Phys. Rev. D55 (1997) 1.

[8] R. Amorim and A. Das, Mod. Phys. Lett. A9 (1994) 3543; R. Amorim, Z. Phys. C67 (1995) 695.

[9] W. T. Kim, Y.-W. Kim, M.-I. Park, Y.-J. Park and S. J. Yoon, J. Phys. G: Nucl. Part. 23 (1997) 325; Y.-W. Kim, M.-I. Park, Y.-J. Park and S. J. Yoon, Int. J. Mod. Phys. A (to be published) (hep-th/9702002).

[10] R. Banerjee and J. Barcelos-Neto, "Hamiltonian embedding of the massive Yang-Mills theory and the generalized Stückelberg formalism", UFRJ preprint (hep-th/9701080);

[11] M.-I. Park and Y.--J. Park, "Quantization of the non-Abelian Proca model based on the improved BFT formalism", SOGANG-HEP 212/97 hepth/9702134).

[12] Y.-W. Kim and K.D. Rothe, "BFT Hamiltonian Embedding of non-abelian self-dual model", HD-THEP-97-25 (hep-th/9706018).

[13] O. Babelon, F. A. Schaposnik and C. M. Viallet, Phys. Lett. B177 (1986) 385.

[14] K. Harada and I. Tsutsui, Phys. Lett. B183 (1987) 311.

[15] E. Abdalla, M. C. B. Abdalla and K. D. Rothe, "Non-perturbative methods in two-dimensional Quantum Field Theory", World Scientific, Singapore, 1991.

[16] E. S. Fradkin and G. A. Vilkovisky, Phys. Lett. B55 (1975) 224; M. Henneaux, Phys. Rep. C126 (1985) 1. 